\title{
Enhanced LSTM for Natural Language Inference
}

\author{
Qian Chen \\ University of Science and \\ Technology of China \\ cq1231@mail.ustc . edu. cn
}

Zhenhua Ling

University of Science and

Technology of China

zhling@ustc.edu.cn

\author{
Hui Jiang \\ York University \\ hjecse.yorku.ca
}

\author{
Xiaodan Zhu \\ National Research Council Canada \\ xiaodan.zhu@nrc-cnrc.gc.ca \\ Xiaodan Zhu \\ gc. ca
anada
}

\begin{abstract}
Reasoning and inference are central to human and artificial intelligence. Modeling inference in human language is very challenging. With the availability of large annotated data (Bowman et al., 2015), it has recently become feasible to train neural network based inference models, which have shown to be very effective. In this paper, we present a new state-of-the-art result, achieving the accuracy of $88.6 \%$ on the Stanford Natural Language Inference Dataset. Unlike the previous top models that use very complicated network architectures, we first demonstrate that carefully designing sequential inference models based on chain LSTMs can outperform all previous models. Based on this, we further show that by explicitly considering recursive architectures in both local inference modeling and inference composition, we achieve additional improvement. Particularly, incorporating syntactic parsing information contributes to our best result-it further improves the performance even when added to the already very strong model.
\end{abstract}

\section{Introduction}

Reasoning and inference are central to both human and artificial intelligence. Modeling inference in human language is notoriously challenging but is a basic problem towards true natural language understanding, as pointed out by MacCartney and Manning (2008), “a necessary (if not sufficient)

\author{
Diana Inkpen \\ University of Ottawa \\ dianalsite.uottawa.ca
}

condition for true natural language understanding is a mastery of open-domain natural language inference." The previous work has included extensive research on recognizing textual entailment.

Specifically, natural language inference (NLI) is concerned with determining whether a naturallanguage hypothesis $h$ can be inferred from a premise $p$, as depicted in the following example from MacCartney (2009), where the hypothesis is regarded to be entailed from the premise.

p: Several airlines polled saw costs grow more than expected, even after adjusting for inflation.

$\mathrm{h}$ : Some of the companies in the poll reported cost increases.

The most recent years have seen advances in modeling natural language inference. An important contribution is the creation of a much larger annotated dataset, the Stanford Natural Language Inference (SNLI) dataset (Bowman et al., 2015). The corpus has 570,000 human-written English sentence pairs manually labeled by multiple human subjects. This makes it feasible to train more complex inference models. Neural network models, which often need relatively large annotated data to estimate their parameters, have shown to achieve the state of the art on SNLI (Bowman et al., 2015, 2016; Munkhdalai and Yu, 2016b; Parikh et al., 2016; Sha et al., 2016; Paria et al., 2016).

While some previous top-performing models use rather complicated network architectures to achieve the state-of-the-art results (Munkhdalai and Yu, 2016b), we demonstrate in this paper that enhancing sequential inference models based on chain 
models can outperform all previous results, suggesting that the potentials of such sequential inference approaches have not been fully exploited yet. More specifically, we show that our sequential inference model achieves an accuracy of $88.0 \%$ on the SNLI benchmark.

Exploring syntax for NLI is very attractive to us. In many problems, syntax and semantics interact closely, including in semantic composition (Partee, 1995), among others. Complicated tasks such as natural language inference could well involve both, which has been discussed in the context of recognizing textual entailment (RTE) (Mehdad et al., 2010; Ferrone and Zanzotto, 2014). In this paper, we are interested in exploring this within the neural network frameworks, with the presence of relatively large training data. We show that by explicitly encoding parsing information with recursive networks in both local inference modeling and inference composition and by incorporating it into our framework, we achieve additional improvement, increasing the performance to a new state of the art with an $88.6 \%$ accuracy.

\section{Related Work}

Early work on natural language inference has been performed on rather small datasets with more conventional methods (refer to MacCartney (2009) for a good literature survey), which includes a large bulk of work on recognizing textual entailment, such as (Dagan et al., 2005; Iftene and Balahur-Dobrescu, 2007), among others. More recently, Bowman et al. (2015) made available the SNLI dataset with 570,000 human annotated sentence pairs. They also experimented with simple classification models as well as simple neural networks that encode the premise and hypothesis independently. Rocktäschel et al. (2015) proposed neural attention-based models for NLI, which captured the attention information. In general, attention based models have been shown to be effective in a wide range of tasks, including machine translation (Bahdanau et al., 2014), speech recognition (Chorowski et al., 2015; Chan et al., 2016), image caption (Xu et al., 2015), and text summarization (Rush et al., 2015; Chen et al., 2016), among others. For NLI, the idea allows neural models to pay attention to specific areas of the sentences.

A variety of more advanced networks have been developed since then (Bowman et al., 2016; Vendrov et al., 2015; Mou et al., 2016; Liu et al., 2016;
Munkhdalai and Yu, 2016a; Rocktäschel et al., 2015; Wang and Jiang, 2016; Cheng et al., 2016; Parikh et al., 2016; Munkhdalai and Yu, 2016b; Sha et al., 2016; Paria et al., 2016). Among them, more relevant to ours are the approaches proposed by Parikh et al. (2016) and Munkhdalai and Yu (2016b), which are among the best performing models.

Parikh et al. (2016) propose a relatively simple but very effective decomposable model. The model decomposes the NLI problem into subproblems that can be solved separately. On the other hand, Munkhdalai and Yu (2016b) propose much more complicated networks that consider sequential LSTM-based encoding, recursive networks, and complicated combinations of attention models, which provide about $0.5 \%$ gain over the results reported by Parikh et al. (2016).

It is, however, not very clear if the potential of the sequential inference networks has been well exploited for NLI. In this paper, we first revisit this problem and show that enhancing sequential inference models based on chain networks can actually outperform all previous results. We further show that explicitly considering recursive architectures to encode syntactic parsing information for NLI could further improve the performance.

\section{Hybrid Neural Inference Models}

We present here our natural language inference networks which are composed of the following major components: input encoding, local inference modeling, and inference composition. Figure 1 shows a high-level view of the architecture. Vertically, the figure depicts the three major components, and horizontally, the left side of the figure represents our sequential NLI model named ESIM, and the right side represents networks that incorporate syntactic parsing information in tree LSTMs.

In our notation, we have two sentences $\mathbf{a}=$ $\left(\mathbf{a}_{1}, \ldots, \mathbf{a}_{\ell_{a}}\right)$ and $\mathbf{b}=\left(\mathbf{b}_{1}, \ldots, \mathbf{b}_{\ell_{b}}\right)$, where $\mathbf{a}$ is $\mathbf{a}$ premise and $\mathbf{b}$ a hypothesis. The $\mathbf{a}_{i}$ or $\mathbf{b}_{j} \in \mathbb{R}^{l}$ is an embedding of $l$-dimensional vector, which can be initialized with some pre-trained word embeddings and organized with parse trees. The goal is to predict a label $y$ that indicates the logic relationship between $\mathbf{a}$ and $\mathbf{b}$.

\subsection{Input Encoding}

We employ bidirectional LSTM (BiLSTM) as one of our basic building blocks for NLI. We first use it 


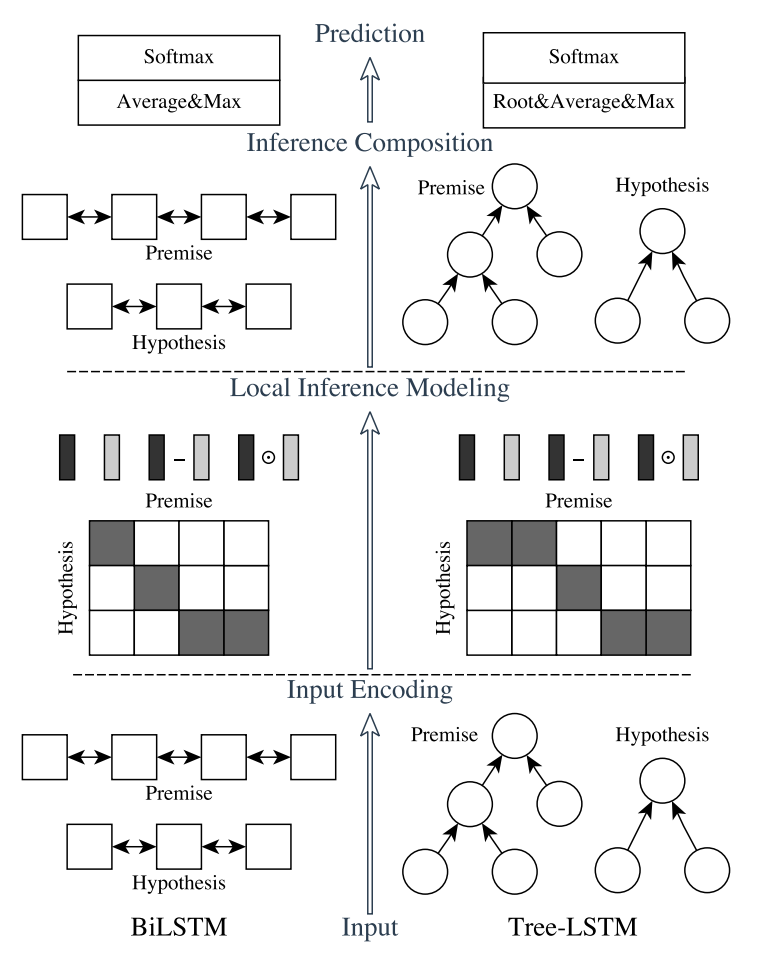

Figure 1: A high-level view of our hybrid neural inference networks.

to encode the input premise and hypothesis (Equation (1) and (2)). Here BiLSTM learns to represent a word (e.g., $\mathbf{a}_{i}$ ) and its context. Later we will also use BiLSTM to perform inference composition to construct the final prediction, where BiLSTM encodes local inference information and its interaction. To bookkeep the notations for later use, we write as $\overline{\mathbf{a}}_{i}$ the hidden (output) state generated by the BiLSTM at time $i$ over the input sequence a. The same is applied to $\overline{\mathbf{b}}_{j}$ :

$$
\begin{aligned}
\overline{\mathbf{a}}_{i} & =\operatorname{BiLSTM}(\mathbf{a}, i), \forall i \in\left[1, \ldots, \ell_{a}\right], \\
\overline{\mathbf{b}}_{j} & =\operatorname{BiLSTM}(\mathbf{b}, j), \forall j \in\left[1, \ldots, \ell_{b}\right] .
\end{aligned}
$$

Due to the space limit, we will skip the description of the basic chain LSTM and readers can refer to Hochreiter and Schmidhuber (1997) for details. Briefly, when modeling a sequence, an LSTM employs a set of soft gates together with a memory cell to control message flows, resulting in an effective modeling of tracking long-distance information/dependencies in a sequence.

A bidirectional LSTM runs a forward and backward LSTM on a sequence starting from the left and the right end, respectively. The hidden states generated by these two LSTMs at each time step are concatenated to represent that time step and its context. Note that we used LSTM memory blocks in our models. We examined other recurrent memory blocks such as GRUs (Gated Recurrent Units) (Cho et al., 2014) and they are inferior to LSTMs on the heldout set for our NLI task.

As discussed above, it is intriguing to explore the effectiveness of syntax for natural language inference; for example, whether it is useful even when incorporated into the best-performing models. To this end, we will also encode syntactic parse trees of a premise and hypothesis through treeLSTM (Zhu et al., 2015; Tai et al., 2015; Le and Zuidema, 2015), which extends the chain LSTM to a recursive network (Socher et al., 2011).

Specifically, given the parse of a premise or hypothesis, a tree node is deployed with a tree-LSTM memory block depicted as in Figure 2 and computed with Equations (3-10). In short, at each node, an input vector $\mathbf{x}_{t}$ and the hidden vectors of its two children (the left child $\mathbf{h}_{t-1}^{L}$ and the right $\mathbf{h}_{t-1}^{R}$ ) are taken in as the input to calculate the current node's hidden vector $\mathbf{h}_{t}$.

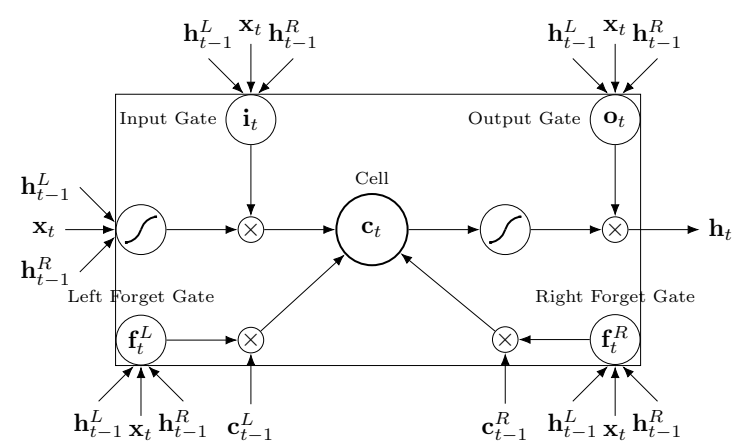

Figure 2: A tree-LSTM memory block.

We describe the updating of a node at a high level with Equation (3) to facilitate references later in the paper, and the detailed computation is described in (4-10). Specifically, the input of a node is used to configure four gates: the input gate $\mathbf{i}_{t}$, output gate $\mathbf{o}_{t}$, and the two forget gates $\mathbf{f}_{t}^{L}$ and $\mathbf{f}_{t}^{R}$. The memory cell $\mathbf{c}_{t}$ considers each child's cell vector, $\mathbf{c}_{t-1}^{L}$ and $\mathbf{c}_{t-1}^{R}$, which are gated by the left forget 
gate $\mathbf{f}_{t}^{L}$ and right forget gate $\mathbf{f}_{t}^{R}$, respectively.

$$
\begin{aligned}
\mathbf{h}_{t} & =\operatorname{TrLSTM}\left(\mathbf{x}_{t}, \mathbf{h}_{t-1}^{L}, \mathbf{h}_{t-1}^{R}\right), \\
\mathbf{h}_{t} & =\mathbf{o}_{t} \odot \tanh \left(\mathbf{c}_{t}\right), \\
\mathbf{o}_{t} & =\sigma\left(\mathbf{W}_{o} \mathbf{x}_{t}+\mathbf{U}_{o}^{L} \mathbf{h}_{t-1}^{L}+\mathbf{U}_{o}^{R} \mathbf{h}_{t-1}^{R}\right), \\
\mathbf{c}_{t} & =\mathbf{f}_{t}^{L} \odot \mathbf{c}_{t-1}^{L}+\mathbf{f}_{t}^{R} \odot \mathbf{c}_{t-1}^{R}+\mathbf{i}_{t} \odot \mathbf{u}_{t}, \\
\mathbf{f}_{t}^{L} & =\sigma\left(\mathbf{W}_{f} \mathbf{x}_{t}+\mathbf{U}_{f}^{L L} \mathbf{h}_{t-1}^{L}+\mathbf{U}_{f}^{L R} \mathbf{h}_{t-1}^{R}\right), \\
\mathbf{f}_{t}^{R} & =\sigma\left(\mathbf{W}_{f} \mathbf{x}_{t}+\mathbf{U}_{f}^{R L} \mathbf{h}_{t-1}^{L}+\mathbf{U}_{f}^{R R} \mathbf{h}_{t-1}^{R}\right), \\
\mathbf{i}_{t} & =\sigma\left(\mathbf{W}_{i} \mathbf{x}_{t}+\mathbf{U}_{i}^{L} \mathbf{h}_{t-1}^{L}+\mathbf{U}_{i}^{R} \mathbf{h}_{t-1}^{R}\right), \\
\mathbf{u}_{t} & =\tanh \left(\mathbf{W}_{c} \mathbf{x}_{t}+\mathbf{U}_{c}^{L} \mathbf{h}_{t-1}^{L}+\mathbf{U}_{c}^{R} \mathbf{h}_{t-1}^{R}\right),
\end{aligned}
$$

where $\sigma$ is the sigmoid function, $\odot$ is the elementwise multiplication of two vectors, and all $\mathbf{W} \in$ $\mathbb{R}^{d \times l}, \mathbf{U} \in \mathbb{R}^{d \times d}$ are weight matrices to be learned.

In the current input encoding layer, $\mathbf{x}_{t}$ is used to encode a word embedding for a leaf node. Since a non-leaf node does not correspond to a specific word, we use a special vector $\mathbf{x}_{t}^{\prime}$ as its input, which is like an unknown word. However, in the inference composition layer that we discuss later, the goal of using tree-LSTM is very different; the input $\mathbf{x}_{t}$ will be very different as well-it will encode local inference information and will have values at all tree nodes.

\subsection{Local Inference Modeling}

Modeling local subsentential inference between a premise and hypothesis is the basic component for determining the overall inference between these two statements. To closely examine local inference, we explore both the sequential and syntactic tree models that have been discussed above. The former helps collect local inference for words and their context, and the tree LSTM helps collect local information between (linguistic) phrases and clauses.

Locality of inference Modeling local inference needs to employ some forms of hard or soft alignment to associate the relevant subcomponents between a premise and a hypothesis. This includes early methods motivated from the alignment in conventional automatic machine translation (MacCartney, 2009). In neural network models, this is often achieved with soft attention.

Parikh et al. (2016) decomposed this process: the word sequence of the premise (or hypothesis) is regarded as a bag-of-word embedding vector and inter-sentence "alignment" (or attention) is computed individually to softly align each word to the content of hypothesis (or premise, respectively). While their basic framework is very effective, achieving one of the previous best results, using a pre-trained word embedding by itself does not automatically consider the context around a word in NLI. Parikh et al. (2016) did take into account the word order and context information through an optional distance-sensitive intra-sentence attention.

In this paper, we argue for leveraging attention over the bidirectional sequential encoding of the input, as discussed above. We will show that this plays an important role in achieving our best results, and the intra-sentence attention used by Parikh et al. (2016) actually does not further improve over our model, while the overall framework they proposed is very effective.

Our soft alignment layer computes the attention weights as the similarity of a hidden state tuple $<\overline{\mathbf{a}}_{i}, \overline{\mathbf{b}}_{j}>$ between a premise and a hypothesis with Equation (11). We did study more complicated relationships between $\overline{\mathbf{a}}_{i}$ and $\overline{\mathbf{b}}_{j}$ with multilayer perceptrons, but observed no further improvement on the heldout data.

$$
e_{i j}=\overline{\mathbf{a}}_{i}^{T} \overline{\mathbf{b}}_{j}
$$

In the formula, $\overline{\mathbf{a}}_{i}$ and $\overline{\mathbf{b}}_{j}$ are computed earlier in Equations (1) and (2), or with Equation (3) when tree-LSTM is used. Again, as discussed above, we will use bidirectional LSTM and tree-LSTM to encode the premise and hypothesis, respectively. In our sequential inference model, unlike in Parikh et al. (2016) which proposed to use a function $F\left(\overline{\mathbf{a}}_{i}\right)$, i.e., a feedforward neural network, to map the original word representation for calculating $e_{i j}$, we instead advocate to use BiLSTM, which encodes the information in premise and hypothesis very well and achieves better performance shown in the experiment section. We tried to apply the $F($.) function on our hidden states before computing $e_{i j}$ and it did not further help our models.

Local inference collected over sequences Local inference is determined by the attention weight $e_{i j}$ computed above, which is used to obtain the local relevance between a premise and hypothesis. For the hidden state of a word in a premise, i.e., $\overline{\mathbf{a}}_{i}$ (already encoding the word itself and its context), the relevant semantics in the hypothesis is identified and composed using $e_{i j}$, more specifically 
with Equation (12).

$$
\begin{aligned}
\tilde{\mathbf{a}}_{i} & =\sum_{j=1}^{\ell_{b}} \frac{\exp \left(e_{i j}\right)}{\sum_{k=1}^{\ell_{b}} \exp \left(e_{i k}\right)} \overline{\mathbf{b}}_{j}, \forall i \in\left[1, \ldots, \ell_{a}\right], \\
\tilde{\mathbf{b}}_{j} & =\sum_{i=1}^{\ell_{a}} \frac{\exp \left(e_{i j}\right)}{\sum_{k=1}^{\ell_{a}} \exp \left(e_{k j}\right)} \overline{\mathbf{a}}_{i}, \forall j \in\left[1, \ldots, \ell_{b}\right],
\end{aligned}
$$

where $\tilde{\mathbf{a}}_{i}$ is a weighted summation of $\left\{\overline{\mathbf{b}}_{j}\right\}_{j=1}^{\ell_{b}}$. Intuitively, the content in $\left\{\overline{\mathbf{b}}_{j}\right\}_{j=1}^{\ell_{b}}$ that is relevant to $\overline{\mathbf{a}}_{i}$ will be selected and represented as $\tilde{\mathbf{a}}_{i}$. The same is performed for each word in the hypothesis with Equation (13).

Local inference collected over parse trees We use tree models to help collect local inference information over linguistic phrases and clauses in this layer. The tree structures of the premise and hypothesis are produced by a constituency parser.

Once the hidden states of a tree are all computed with Equation (3), we treat all tree nodes equally as we do not have further heuristics to discriminate them, but leave the attention weights to figure out their relationship. So, we use Equation (11) to compute the attention weights for all node pairs between a premise and hypothesis. This connects all words, constituent phrases, and clauses between the premise and hypothesis. We then collect the information between all the pairs with Equations (12) and (13) and feed them into the next layer.

Enhancement of local inference information In our models, we further enhance the local inference information collected. We compute the difference and the element-wise product for the tuple $<\overline{\mathbf{a}}, \tilde{\mathbf{a}}>$ as well as for $\langle\overline{\mathbf{b}}, \tilde{\mathbf{b}}\rangle$. We expect that such operations could help sharpen local inference information between elements in the tuples and capture inference relationships such as contradiction. The difference and element-wise product are then concatenated with the original vectors, $\overline{\mathbf{a}}$ and $\tilde{\mathbf{a}}$, or $\overline{\mathbf{b}}$ and $\tilde{\mathbf{b}}$, respectively (Mou et al., 2016; Zhang et al., 2017). The enhancement is performed for both the sequential and the tree models.

$$
\begin{aligned}
\mathbf{m}_{a} & =[\overline{\mathbf{a}} ; \tilde{\mathbf{a}} ; \overline{\mathbf{a}}-\tilde{\mathbf{a}} ; \overline{\mathbf{a}} \odot \tilde{\mathbf{a}}], \\
\mathbf{m}_{b} & =[\overline{\mathbf{b}} ; \tilde{\mathbf{b}} ; \overline{\mathbf{b}}-\tilde{\mathbf{b}} ; \overline{\mathbf{b}} \odot \tilde{\mathbf{b}}] .
\end{aligned}
$$

This process could be regarded as a special case of modeling some high-order interaction between the tuple elements. Along this direction, we have also further modeled the interaction by feeding the tuples into feedforward neural networks and added the top layer hidden states to the above concatenation. We found that it does not further help the inference accuracy on the heldout dataset.

\subsection{Inference Composition}

To determine the overall inference relationship between a premise and hypothesis, we explore a composition layer to compose the enhanced local inference information $\mathbf{m}_{a}$ and $\mathbf{m}_{b}$. We perform the composition sequentially or in its parse context using BiLSTM and tree-LSTM, respectively.

The composition layer In our sequential inference model, we keep using BiLSTM to compose local inference information sequentially. The formulas for BiLSTM are similar to those in Equations (1) and (2) in their forms so we skip the details, but the aim is very different here- they are used to capture local inference information $\mathbf{m}_{a}$ and $\mathbf{m}_{b}$ and their context here for inference composition.

In the tree composition, the high-level formulas of how a tree node is updated to compose local inference is as follows:

$$
\begin{aligned}
& \mathbf{v}_{a, t}=\operatorname{Tr} \operatorname{LSTM}\left(F\left(\mathbf{m}_{a, t}\right), \mathbf{h}_{t-1}^{L}, \mathbf{h}_{t-1}^{R}\right), \\
& \mathbf{v}_{b, t}=\operatorname{TrLSTM}\left(F\left(\mathbf{m}_{b, t}\right), \mathbf{h}_{t-1}^{L}, \mathbf{h}_{t-1}^{R}\right) .
\end{aligned}
$$

We propose to control model complexity in this layer, since the concatenation we described above to compute $\mathbf{m}_{a}$ and $\mathbf{m}_{b}$ can significantly increase the overall parameter size to potentially overfit the models. We propose to use a mapping $F$ as in Equation (16) and (17). More specifically, we use a 1-layer feedforward neural network with the ReLU activation. This function is also applied to BiLSTM in our sequential inference composition.

Pooling Our inference model converts the resulting vectors obtained above to a fixed-length vector with pooling and feeds it to the final classifier to determine the overall inference relationship.

We consider that summation (Parikh et al., 2016) could be sensitive to the sequence length and hence less robust. We instead suggest the following strategy: compute both average and max pooling, and concatenate all these vectors to form the final fixed length vector v. Our experiments show that this leads to significantly better results than summation. The final fixed length vector $\mathbf{v}$ is calculated 
as follows:

$$
\begin{aligned}
\mathbf{v}_{a, \text { ave }} & =\sum_{i=1}^{\ell_{a}} \frac{\mathbf{v}_{a, i}}{\ell_{a}}, \quad \mathbf{v}_{a, \max }=\max _{i=1}^{\ell_{a}} \mathbf{v}_{a, i}, \\
\mathbf{v}_{b, \text { ave }} & =\sum_{j=1}^{\ell_{b}} \frac{\mathbf{v}_{b, j}}{\ell_{b}}, \quad \mathbf{v}_{b, \text { max }}=\max _{j=1}^{\ell_{b}} \mathbf{v}_{b, j}, \\
\mathbf{v} & =\left[\mathbf{v}_{a, \text { ave }} ; \mathbf{v}_{a, \max } ; \mathbf{v}_{b, \text { ave }} ; \mathbf{v}_{b, \max }\right] .
\end{aligned}
$$

Note that for tree composition, Equation (20) is slightly different from that in sequential composition. Our tree composition will concatenate also the hidden states computed for the roots with Equations (16) and (17), which are not shown here.

We then put $\mathbf{v}$ into a final multilayer perceptron (MLP) classifier. The MLP has a hidden layer with tanh activation and softmax output layer in our experiments. The entire model (all three components described above) is trained end-to-end. For training, we use multi-class cross-entropy loss.

Overall inference models Our model can be based only on the sequential networks by removing all tree components and we call it Enhanced Sequential Inference Model (ESIM) (see the left part of Figure 1). We will show that ESIM outperforms all previous results. We will also encode parse information with tree LSTMs in multiple layers as described (see the right side of Figure 1). We train this model and incorporate it into ESIM by averaging the predicted probabilities to get the final label for a premise-hypothesis pair. We will show that parsing information complements very well with ESIM and further improves the performance, and we call the final model Hybrid Inference Model (HIM).

\section{Experimental Setup}

Data The Stanford Natural Language Inference (SNLI) corpus (Bowman et al., 2015) focuses on three basic relationships between a premise and a potential hypothesis: the premise entails the hypothesis (entailment), they contradict each other (contradiction), or they are not related (neutral). The original SNLI corpus contains also "the other" category, which includes the sentence pairs lacking consensus among multiple human annotators. As in the related work, we remove this category. We used the same split as in Bowman et al. (2015) and other previous work.
The parse trees used in this paper are produced by the Stanford PCFG Parser 3.5.3 (Klein and Manning, 2003) and they are delivered as part of the SNLI corpus. We use classification accuracy as the evaluation metric, as in related work.

Training We use the development set to select models for testing. To help replicate our results, we publish our code ${ }^{1}$. Below, we list our training details. We use the Adam method (Kingma and $\mathrm{Ba}, 2014)$ for optimization. The first momentum is set to be 0.9 and the second 0.999 . The initial learning rate is 0.0004 and the batch size is 32 . All hidden states of LSTMs, tree-LSTMs, and word embeddings have 300 dimensions.

We use dropout with a rate of 0.5 , which is applied to all feedforward connections. We use pre-trained 300-D Glove 840B vectors (Pennington et al., 2014) to initialize our word embeddings. Out-of-vocabulary (OOV) words are initialized randomly with Gaussian samples. All vectors including word embedding are updated during training.

\section{Results}

Overall performance Table 1 shows the results of different models. The first row is a baseline classifier presented by Bowman et al. (2015) that considers handcrafted features such as BLEU score of the hypothesis with respect to the premise, the overlapped words, and the length difference between them, etc.

The next group of models (2)-(7) are based on sentence encoding. The model of Bowman et al. (2016) encodes the premise and hypothesis with two different LSTMs. The model in Vendrov et al. (2015) uses unsupervised "skip-thoughts" pre-training in GRU encoders. The approach proposed by Mou et al. (2016) considers tree-based CNN to capture sentence-level semantics, while the model of Bowman et al. (2016) introduces a stack-augmented parser-interpreter neural network (SPINN) which combines parsing and interpretation within a single tree-sequence hybrid model. The work by Liu et al. (2016) uses BiLSTM to generate sentence representations, and then replaces average pooling with intra-attention. The approach proposed by Munkhdalai and Yu (2016a) presents a memory augmented neural network, neural semantic encoders (NSE), to encode sentences.

The next group of methods in the table, models

\footnotetext{
${ }^{1}$ https://github.com/lukecq1231/nli
} 


\begin{tabular}{lccc}
\hline Model & \#Para. & Train & Test \\
\hline (1) Handcrafted features (Bowman et al., 2015) & - & 99.7 & 78.2 \\
\hline (2) 300D LSTM encoders (Bowman et al., 2016) & $3.0 \mathrm{M}$ & 83.9 & 80.6 \\
(3) 1024D pretrained GRU encoders (Vendrov et al., 2015) & $15 \mathrm{M}$ & 98.8 & 81.4 \\
(4) 300D tree-based CNN encoders (Mou et al., 2016) & $3.5 \mathrm{M}$ & 83.3 & 82.1 \\
(5) 300D SPINN-PI encoders (Bowman et al., 2016) & $3.7 \mathrm{M}$ & 89.2 & 83.2 \\
(6) 600D BiLSTM intra-attention encoders (Liu et al., 2016) & $2.8 \mathrm{M}$ & 84.5 & 84.2 \\
(7) 300D NSE encoders (Munkhdalai and Yu, 2016a) & $3.0 \mathrm{M}$ & 86.2 & 84.6 \\
\hline (8) 100D LSTM with attention (Rocktäschel et al., 2015) & $250 \mathrm{~K}$ & 85.3 & 83.5 \\
(9) 300D mLSTM (Wang and Jiang, 2016) & $1.9 \mathrm{M}$ & 92.0 & 86.1 \\
(10) 450D LSTMN with deep attention fusion (Cheng et al., 2016) & $3.4 \mathrm{M}$ & 88.5 & 86.3 \\
(11) 200D decomposable attention model (Parikh et al., 2016) & $380 \mathrm{~K}$ & 89.5 & 86.3 \\
(12) Intra-sentence attention + (11) (Parikh et al., 2016) & $580 \mathrm{~K}$ & 90.5 & 86.8 \\
(13) 300D NTI-SLSTM-LSTM (Munkhdalai and Yu, 2016b) & $3.2 \mathrm{M}$ & 88.5 & 87.3 \\
(14) 300D re-read LSTM (Sha et al., 2016) & $2.0 \mathrm{M}$ & 90.7 & 87.5 \\
(15) 300D btree-LSTM encoders (Paria et al., 2016) & $2.0 \mathrm{M}$ & 88.6 & 87.6 \\
\hline (16) 600D ESIM & $4.3 \mathrm{M}$ & 92.6 & $\underline{88.0}$ \\
(17) HIM (600D ESIM + 300D Syntactic tree-LSTM) & $7.7 \mathrm{M}$ & 93.5 & $\mathbf{8 8 . 6}$ \\
\hline
\end{tabular}

Table 1: Accuracies of the models on SNLI. Our final model achieves the accuracy of $88.6 \%$, the best result observed on SNLI, while our enhanced sequential encoding model attains an accuracy of $88.0 \%$, which also outperform the previous models.

(8)-(15), are inter-sentence attention-based model. The model marked with Rocktäschel et al. (2015) is LSTMs enforcing the so called word-by-word attention. The model of Wang and Jiang (2016) extends this idea to explicitly enforce word-by-word matching between the hypothesis and the premise. Long short-term memory-networks (LSTMN) with deep attention fusion (Cheng et al., 2016) link the current word to previous words stored in memory. Parikh et al. (2016) proposed a decomposable attention model without relying on any word-order information. In general, adding intra-sentence attention yields further improvement, which is not very surprising as it could help align the relevant text spans between premise and hypothesis. The model of Munkhdalai and Yu (2016b) extends the framework of Wang and Jiang (2016) to a full n-ary tree model and achieves further improvement. Sha et al. (2016) proposes a special LSTM variant which considers the attention vector of another sentence as an inner state of LSTM. Paria et al. (2016) use a neural architecture with a complete binary tree-LSTM encoders without syntactic information.

The table shows that our ESIM model achieves an accuracy of $88.0 \%$, which has already outperformed all the previous models, including those using much more complicated network architectures (Munkhdalai and Yu, 2016b).
We ensemble our ESIM model with syntactic tree-LSTMs (Zhu et al., 2015) based on syntactic parse trees and achieve significant improvement over our best sequential encoding model ESIM, attaining an accuracy of $88.6 \%$. This shows that syntactic tree-LSTMs complement well with ESIM.

\begin{tabular}{lcc}
\hline Model & Train & Test \\
\hline (17) HIM (ESIM + syn.tree) & 93.5 & 88.6 \\
(18) ESIM + tree & 91.9 & 88.2 \\
(16) ESIM & 92.6 & 88.0 \\
(19) ESIM - ave./max & 92.9 & 87.1 \\
(20) ESIM - diff./prod. & 91.5 & 87.0 \\
(21) ESIM - inference BiLSTM & 91.3 & 87.3 \\
(22) ESIM - encoding BiLSTM & 88.7 & 86.3 \\
(23) ESIM - P-based attention & 91.6 & 87.2 \\
(24) ESIM - H-based attention & 91.4 & 86.5 \\
(25) syn.tree & 92.9 & 87.8 \\
\hline
\end{tabular}

Table 2: Ablation performance of the models.

Ablation analysis We further analyze the major components that are of importance to help us achieve good performance. From the best model, we first replace the syntactic tree-LSTM with the full tree-LSTM without encoding syntactic parse information. More specifically, two adjacent words in a sentence are merged to form a parent node, and 


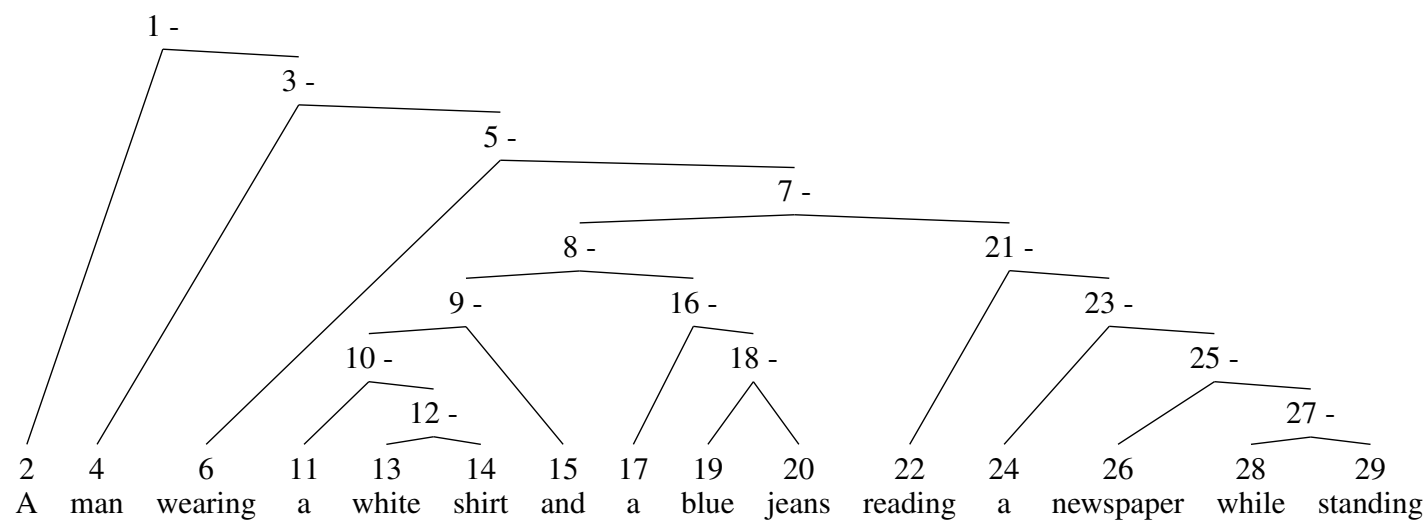

(a) Binarized constituency tree of premise

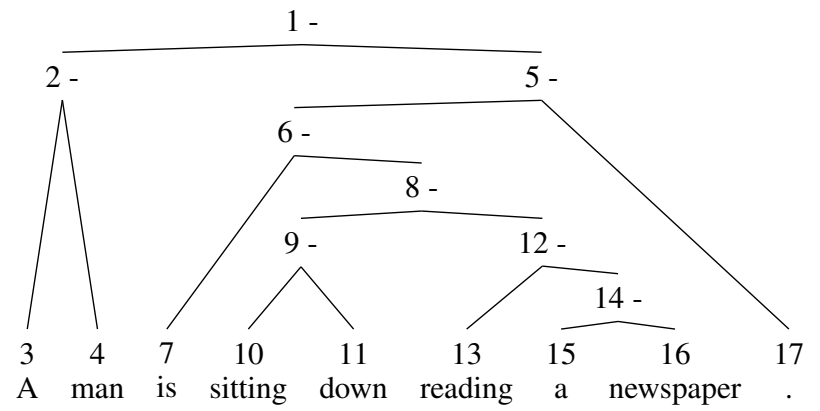

(b) Binarized constituency tree of hypothesis

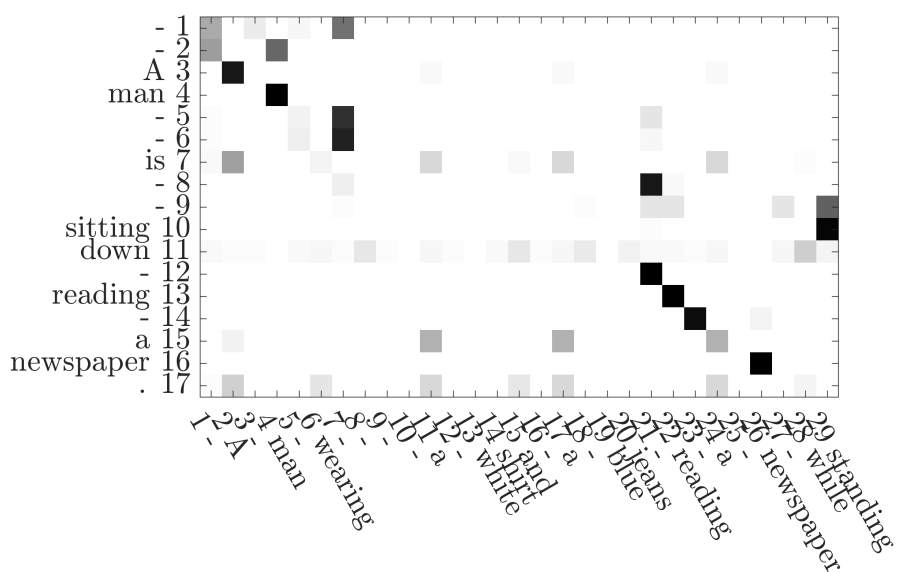

(c) Normalized attention weights of tree-LSTM

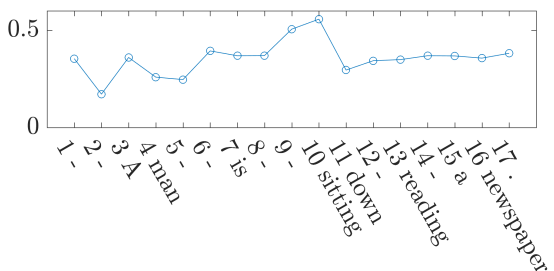

(d) Input gate of tree-LSTM in inference composition $\left(l^{2}\right.$-norm)

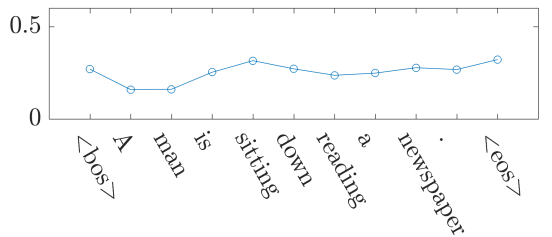

(e) Input gate of BiLSTM in inference composition $\left(l^{2}\right.$-norm)

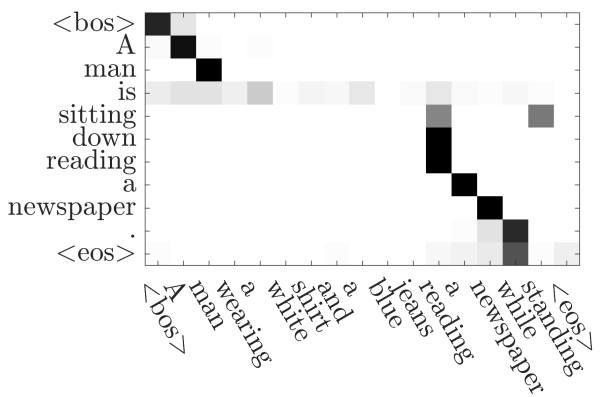

(f) Normalized attention weights of BiLSTM

Figure 3: An example for analysis. Subfigures (a) and (b) are the constituency parse trees of the premise and hypothesis, respectively. "-" means a non-leaf or a null node. Subfigures (c) and (f) are attention visualization of the tree model and ESIM, respectively. The darker the color, the greater the value. The premise is on the $\mathrm{x}$-axis and the hypothesis is on y-axis. Subfigures (d) and (e) are input gates' $l^{2}$-norm of tree-LSTM and BiLSTM in inference composition, respectively.

this process continues and results in a full binary tree, where padding nodes are inserted when there are no enough leaves to form a full tree. Each tree node is implemented with a tree-LSTM block (Zhu et al., 2015) same as in model (17). Table 2 shows that with this replacement, the performance drops to $88.2 \%$.

Furthermore, we note the importance of the layer performing the enhancement for local inference information in Section 3.2 and the pooling layer in inference composition in Section 3.3. Table 2 suggests that the NLI task seems very sensitive to the 
layers. If we remove the pooling layer in inference composition and replace it with summation as in Parikh et al. (2016), the accuracy drops to $87.1 \%$. If we remove the difference and elementwise product from the local inference enhancement layer, the accuracy drops to $87.0 \%$. To provide some detailed comparison with Parikh et al. (2016), replacing bidirectional LSTMs in inference composition and also input encoding with feedforward neural network reduces the accuracy to $87.3 \%$ and $86.3 \%$ respectively.

The difference between ESIM and each of the other models listed in Table 2 is statistically significant under the one-tailed paired t-test at the $99 \%$ significance level. The difference between model (17) and (18) is also significant at the same level. Note that we cannot perform significance test between our models with the other models listed in Table 1 since we do not have the output of the other models.

If we remove the premise-based attention from ESIM (model 23), the accuracy drops to $87.2 \%$ on the test set. The premise-based attention means when the system reads a word in a premise, it uses soft attention to consider all relevant words in hypothesis. Removing the hypothesis-based attention (model 24) decrease the accuracy to $86.5 \%$, where hypothesis-based attention is the attention performed on the other direction for the sentence pairs. The results show that removing hypothesisbased attention affects the performance of our model more, but removing the attention from the other direction impairs the performance too.

The stand-alone syntactic tree-LSTM model achieves an accuracy of $87.8 \%$, which is comparable to that of ESIM. We also computed the oracle score of merging syntactic tree-LSTM and ESIM, which picks the right answer if either is right. Such an oracle/upper-bound accuracy on test set is $91.7 \%$, which suggests how much tree-LSTM and ESIM could ideally complement each other. As far as the speed is concerned, training tree-LSTM takes about 40 hours on Nvidia-Tesla K40M and ESIM takes about 6 hours, which is easily extended to larger scale of data.

Further analysis We showed that encoding syntactic parsing information helps recognize natural language inference-it additionally improves the strong system. Figure 3 shows an example where tree-LSTM makes a different and correct decision. In subfigure (d), the larger values at the input gates on nodes 9 and 10 indicate that those nodes are important in making the final decision. We observe that in subfigure (c), nodes 9 and 10 are aligned to node 29 in the premise. Such information helps the system decide that this pair is a contradiction. Accordingly, in subfigure (e) of sequential BiLSTM, the words sitting and down do not play an important role for making the final decision. Subfigure (f) shows that sitting is equally aligned with reading and standing and the alignment for word down is not that useful.

\section{Conclusions and Future Work}

We propose neural network models for natural language inference, which achieve the best results reported on the SNLI benchmark. The results are first achieved through our enhanced sequential inference model, which outperformed the previous models, including those employing more complicated network architectures, suggesting that the potential of sequential inference models have not been fully exploited yet. Based on this, we further show that by explicitly considering recursive architectures in both local inference modeling and inference composition, we achieve additional improvement. Particularly, incorporating syntactic parsing information contributes to our best result: it further improves the performance even when added to the already very strong model.

Future work interesting to us includes exploring the usefulness of external resources such as WordNet and contrasting-meaning embedding (Chen et al., 2015) to help increase the coverage of wordlevel inference relations. Modeling negation more closely within neural network frameworks (Socher et al., 2013; Zhu et al., 2014) may help contradiction detection.

\section{Acknowledgments}

The first and the third author of this paper were supported in part by the Science and Technology Development of Anhui Province, China (Grants No. 2014z02006), the Fundamental Research Funds for the Central Universities (Grant No. WK2350000001) and the Strategic Priority Research Program of the Chinese Academy of Sciences (Grant No. XDB02070006). 


\section{References}

Dzmitry Bahdanau, Kyunghyun Cho, and Yoshua Bengio. 2014. Neural machine translation by jointly learning to align and translate. CoRR abs/1409.0473. http://arxiv.org/abs/1409.0473.

Samuel Bowman, Gabor Angeli, Christopher Potts, and D. Christopher Manning. 2015. A large annotated corpus for learning natural language inference. In Proceedings of the 2015 Conference on Empirical Methods in Natural Language Processing. Association for Computational Linguistics, pages 632-642. https://doi.org/10.18653/v1/D15-1075.

Samuel Bowman, Jon Gauthier, Abhinav Rastogi, Raghav Gupta, D. Christopher Manning, and Christopher Potts. 2016. A fast unified model for parsing and sentence understanding. In Proceedings of the 54th Annual Meeting of the Association for Computational Linguistics (Volume 1: Long Papers). Association for Computational Linguistics, pages 1466-1477. https://doi.org/10.18653/v1/P16-1139.

William Chan, Navdeep Jaitly, Quoc V. Le, and Oriol Vinyals. 2016. Listen, attend and spell: A neural network for large vocabulary conversational speech recognition. In 2016 IEEE International Conference on Acoustics, Speech and Signal Processing, ICASSP 2016, Shanghai, China, March 20-25, 2016. IEEE, pages 4960-4964. https://doi.org/10.1109/ICASSP.2016.7472621.

Qian Chen, Xiaodan Zhu, Zhenhua Ling, Si Wei, and Hui Jiang. 2016. Distraction-based neural networks for modeling document. In Subbarao Kambhampati, editor, Proceedings of the Twenty-Fifth International Joint Conference on Artificial Intelligence, IJCAI 2016, New York, NY, USA, 9-15 July 2016. IJCAI/AAAI Press, pages 2754-2760. http://www.ijcai.org/Abstract/16/391.

Zhigang Chen, Wei Lin, Qian Chen, Xiaoping Chen, Si Wei, Hui Jiang, and Xiaodan Zhu. 2015. Revisiting word embedding for contrasting meaning. In Proceedings of the 53rd Annual Meeting of the Association for Computational Linguistics and the 7th International Joint Conference on Natural Language Processing (Volume 1: Long Papers). Association for Computational Linguistics, pages 106-115. https://doi.org/10.3115/v1/P15-1011.

Jianpeng Cheng, Li Dong, and Mirella Lapata. 2016. Long short-term memory-networks for machine reading. In Proceedings of the 2016 Conference on Empirical Methods in Natural Language Processing. Association for Computational Linguistics, pages 551-561. http://aclweb.org/anthology/D16-1053.

Kyunghyun Cho, Bart van Merrienboer, Dzmitry Bahdanau, and Yoshua Bengio. 2014. On the properties of neural machine translation: Encoder-decoder approaches. In Dekai Wu, Marine Carpuat, Xavier Carreras, and Eva Maria Vecchi, editors, Proceedings of SSST@EMNLP 2014, Eighth Workshop on
Syntax, Semantics and Structure in Statistical Translation, Doha, Qatar, 25 October 2014. Association for Computational Linguistics, pages 103 111. http://aclweb.org/anthology/W/W14/W144012.pdf.

Jan Chorowski, Dzmitry Bahdanau, Dmitriy Serdyuk, Kyunghyun Cho, and Yoshua Bengio. 2015. Attention-based models for speech recognition. In Corinna Cortes, Neil D. Lawrence, Daniel D. Lee, Masashi Sugiyama, and Roman Garnett, editors, Advances in Neural Information Processing Systems 28: Annual Conference on Neural Information Processing Systems 2015, December 7-12, 2015, Montreal, Quebec, Canada. pages 577-585. http://papers.nips.cc/paper/5847attention-based-models-for-speech-recognition.

Ido Dagan, Oren Glickman, and Bernardo Magnini. 2005. The PASCAL recognising textual entailment challenge. In Machine Learning Challenges, Evaluating Predictive Uncertainty, Visual Object Classification and Recognizing Textual Entailment, First PASCAL Machine Learning Challenges Workshop, MLCW 2005, Southampton, UK, April 11-13, 2005, Revised Selected Papers. pages 177-190.

Lorenzo Ferrone and Massimo Fabio Zanzotto. 2014. Towards syntax-aware compositional distributional semantic models. In Proceedings of COLING 2014, the 25th International Conference on Computational Linguistics: Technical Papers. Dublin City University and Association for Computational Linguistics, pages 721-730. http://aclweb.org/anthology/C141068.

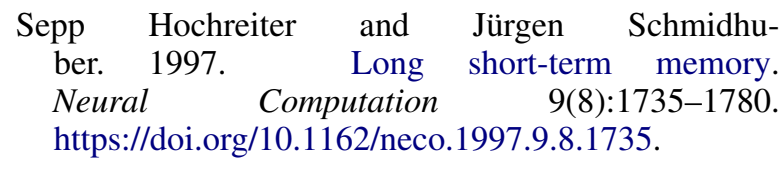

Adrian Iftene and Alexandra Balahur-Dobrescu. 2007. Proceedings of the ACL-PASCAL Workshop on Textual Entailment and Paraphrasing, Association for Computational Linguistics, chapter Hypothesis Transformation and Semantic Variability Rules Used in Recognizing Textual Entailment, pages 125130. http://aclweb.org/anthology/W07-1421.

Diederik P. Kingma and Jimmy Ba. 2014. Adam: A method for stochastic optimization. CoRR abs/1412.6980. http://arxiv.org/abs/1412.6980.

Dan Klein and Christopher D. Manning. 2003. Accurate unlexicalized parsing. In Proceedings of the 41 st Annual Meeting of the Association for Computational Linguistics. http://aclweb.org/anthology/P031054.

Phong Le and Willem Zuidema. 2015. Compositional distributional semantics with long short term memory. In Proceedings of the Fourth Joint Conference on Lexical and Computational Semantics. Association for Computational Linguistics, pages 10-19. https://doi.org/10.18653/v1/S15-1002. 
Yang Liu, Chengjie Sun, Lei Lin, and Xiaolong Wang. 2016. Learning natural language inference using bidirectional LSTM model and inner-attention. CoRR abs/1605.09090. http://arxiv.org/abs/1605.09090.

Bill MacCartney. 2009. Natural Language Inference. Ph.D. thesis, Stanford University.

Bill MacCartney and Christopher D. Manning. 2008. Modeling semantic containment and exclusion in natural language inference. In Proceedings of the 22Nd International Conference on Computational Linguistics - Volume 1. Association for Computational Linguistics, Stroudsburg, PA, USA, COLING '08, pages 521-528. http://dl.acm.org/citation.cfm?id=1599081.1599147.

Yashar Mehdad, Alessandro Moschitti, and Massimo Fabio Zanzotto. 2010. Syntactic/semantic structures for textual entailment recognition. In Human Language Technologies: The 2010 Annual Conference of the North American Chapter of the Association for Computational Linguistics. Association for Computational Linguistics, pages 1020 1028. http://aclweb.org/anthology/N10-1146.

Lili Mou, Rui Men, Ge Li, Yan Xu, Lu Zhang, Rui Yan, and Zhi Jin. 2016. Natural language inference by tree-based convolution and heuristic matching. In Proceedings of the 54th Annual Meeting of the Association for Computational Linguistics (Volume 2: Short Papers). Association for Computational Linguistics, pages 130-136. https://doi.org/10.18653/v1/P16-2022.

Tsendsuren Munkhdalai and Hong Yu. 2016a. Neural semantic encoders. CoRR abs/1607.04315. http://arxiv.org/abs/1607.04315.

Tsendsuren Munkhdalai and Hong Yu. 2016b. Neural tree indexers for text understanding. CoRR abs/1607.04492. http://arxiv.org/abs/1607.04492.

Biswajit Paria, K. M. Annervaz, Ambedkar Dukkipati, Ankush Chatterjee, and Sanjay Podder. 2016. A neural architecture mimicking humans end-to-end for natural language inference. CoRR abs/1611.04741. http://arxiv.org/abs/1611.04741.

Ankur Parikh, Oscar Täckström, Dipanjan Das, and Jakob Uszkoreit. 2016. A decomposable attention model for natural language inference. In Proceedings of the 2016 Conference on Empirical Methods in Natural Language Processing. Association for Computational Linguistics, pages 2249-2255. http://aclweb.org/anthology/D16-1244.

Barbara Partee. 1995. Lexical semantics and compositionality. Invitation to Cognitive Science 1:311-360.

Jeffrey Pennington, Richard Socher, and Christopher Manning. 2014. GloVe: Global vectors for word representation. In Proceedings of the 2014 Conference on Empirical Methods in Natural Language Processing (EMNLP). Association for Computational Linguistics, pages 1532-1543. https://doi.org/10.3115/v1/D14-1162.

Tim Rocktäschel, Edward Grefenstette, Karl Moritz Hermann, Tomás Kociský, and Phil Blunsom. 2015. Reasoning about entailment with neural attention. CoRR abs/1509.06664. http://arxiv.org/abs/1509.06664.

Alexander Rush, Sumit Chopra, and Jason Weston. 2015. A neural attention model for abstractive sentence summarization. In Proceedings of the 2015 Conference on Empirical Methods in Natural Language Processing. Association for Computational Linguistics, pages 379-389. https://doi.org/10.18653/v1/D15-1044.

Lei Sha, Baobao Chang, Zhifang Sui, and Sujian Li. 2016. Reading and thinking: Re-read LSTM unit for textual entailment recognition. In Proceedings of COLING 2016, the 26th International Conference on Computational Linguistics: Technical Papers. The COLING 2016 Organizing Committee, pages 2870-2879. http://aclweb.org/anthology/C161270 .

Richard Socher, Cliff Chiung-Yu Lin, Andrew Y. Ng, and Christopher D. Manning. 2011. Parsing natural scenes and natural language with recursive neural networks. In Lise Getoor and Tobias Scheffer, editors, Proceedings of the 28th International Conference on Machine Learning, ICML 2011, Bellevue, Washington, USA, June 28 - July 2, 2011. Omnipress, pages 129-136.

Richard Socher, Alex Perelygin, Jean Wu, Jason Chuang, D. Christopher Manning, Andrew Ng, and Christopher Potts. 2013. Recursive deep models for semantic compositionality over a sentiment treebank. In Proceedings of the 2013 Conference on Empirical Methods in Natural Language Processing. Association for Computational Linguistics, pages 1631-1642. http://aclweb.org/anthology/D13-1170.

Sheng Kai Tai, Richard Socher, and D. Christopher Manning. 2015. Improved semantic representations from tree-structured long short-term memory networks. In Proceedings of the 53rd Annual Meeting of the Association for Computational Linguistics and the 7th International Joint Conference on Natural Language Processing (Volume 1: Long Papers). Association for Computational Linguistics, pages 1556-1566. https://doi.org/10.3115/v1/P15-1150.

Ivan Vendrov, Ryan Kiros, Sanja Fidler, and Raquel Urtasun. 2015. Order-embeddings of images and language. CoRR abs/1511.06361. http://arxiv.org/abs/1511.06361.

Shuohang Wang and Jing Jiang. 2016. Learning natural language inference with LSTM. In Proceedings of the 2016 Conference of the North American Chapter of the Association for Computational Linguistics: Human Language Technologies. Association for Computational Linguistics, pages 1442 1451. https://doi.org/10.18653/v1/N16-1170. 
Kelvin $\mathrm{Xu}$, Jimmy $\mathrm{Ba}$, Ryan Kiros, Kyunghyun Cho, Aaron C. Courville, Ruslan Salakhutdinov, Richard S. Zemel, and Yoshua Bengio. 2015. Show, attend and tell: Neural image caption generation with visual attention. In Proceedings of the 32nd International Conference on Machine Learning, ICML 2015, Lille, France, 6-11 July 2015. pages 2048-2057. http://jmlr.org/proceedings/papers/v37/xuc15.html.

Junbei Zhang, Xiaodan Zhu, Qian Chen, Lirong Dai, Si Wei, and Hui Jiang. 2017. Exploring question understanding and adaptation in neural-network-based question answering. CoRR abs/arXiv:1703.04617v2. https://arxiv.org/abs/1703.04617.

Xiaodan Zhu, Hongyu Guo, Saif Mohammad, and Svetlana Kiritchenko. 2014. An empirical study on the effect of negation words on sentiment. In Proceedings of the 52nd Annual Meeting of the Association for Computational Linguistics (Volume 1: Long Papers). Association for Computational Linguistics, pages 304-313. https://doi.org/10.3115/v1/P141029.

Xiaodan Zhu, Parinaz Sobhani, and Hongyu Guo. 2015. Long short-term memory over recursive structures. In Proceedings of the 32nd International Conference on Machine Learning, ICML 2015, Lille, France, 6-11 July 2015. pages 1604-1612. http://jmlr.org/proceedings/papers/v37/zhub15.html. 